\title{
ROI Selection Criteria for Finding the Abnormal Tissues from Breast Magnetic Resonance Imaging
}

\author{
Poonam Jaglan, Rajeshwar Dass, Manoj Duhan
}

\begin{abstract}
The imaging methods in breast diagnostics play a preeminent role in the early detection and finding out the exact location \& area of the suspicious breast tissues for malignancy. The further treatment significantly depends on the tumour-tobreast size relationship. The tumor size considered as the most influential factors for pathological/clinical assessment of breast cancer. In general, localization of the tumor's location and also the selection of a region of interest (ROI) were performed manually by an experienced radiologist. The objective of this paper is to propose an effective criterion for selection of ROI for abnormal tissues detection from breast MRI. This paper implements an efficacious ROI selection criterion for finding the exact location \& area of the breast abnormal tissues from magnetic resonance imaging automatically. The proposed algorithm integrates the simple techniques like filtering, edge detection and morphological operations for inner segmentation. Outer breast region segmentation is performed by selecting the peak and valley points and then connects the selected points by applying fit to circle function which makes the MR image rotation invariant. The method is implemented on the 80 images contained in S1 dataset i.e. multi-parametric breast MRI dataset and the evaluation is done through comparative analysis of predicted image with manually segmented images. The experimental results in terms of evaluation matrices i.e. Precision, Recall and Score depict the efficacy of the proposed work.
\end{abstract}

Keywords: Breast MRI, Image segmentation, Region of interest.

\section{INTRODUCTION}

Segmentation is the initial phase for further image analysis. Its main objective is to additionally alter the portrayal of the image which is progressively important and easy to investigate [8]. Generally, noise removal is the pre-processing step followed by segmentation technique to maintain a strategic distance from the false contour determination. The most challenging work is to section the image without any loss of relevant data for restorative diagnosing reason. Image segmentation pursues some particular principles to segment the image into number of regions which further utilized for ROI detection to recognize any lesion or malignancy. The inevitable achievement or disappointment of the investigation or analysis relies upon the segmentation method. Determination of the suspicious area (ROI) is a practically major step in the vigorous analysis of the attributes of a malignant and benign lesion to enhance the specificity.

Revised Manuscript Received on December 30, 2019.

* Correspondence Author

Poonam Jaglan*, Pursuing PhD, Department of Electronics \& Communication Engineering, DCRUST, Murthal, Sonepat, India.

Rajeshwar Dass, Assistant Professor, Department of Electronics \& Communication Engineering, DCRUST, Murthal, Sonepat, India.

Manoj Duhan, Professor, Department of Electronics \& Communication Engineering, DCRUST, Murthal, Sonepat, India.

(c) The Authors. Published by Blue Eyes Intelligence Engineering and Sciences Publication (BEIESP). This is an open access article under the CC BY-NC-ND license (http://creativecommons.org/licenses/by-nc-nd/4.0/)
Eventually, the ROI is selected manually by the radiologist in spite of the fact that there is no accord on the size and ideal location of this.

The selected ROI may be characterized as the group with the highest probability of abnormality. Significant goal of the proposed methodology is to remove the tremendous measure of fatty as well as fibro-glandular tissues from the tumor tissues. The intensity, stage, type, future and treatment of disease must be distinguished based on cancerous cells' orientation/ duct, state of the cell, density of cancerous cells in comparison with normal cells. The paper is additionally arranged as section 3 gives the related work (literature review), section 4 depicts the philanthropy of the proposed work, section 5 demonstrates the performance analysis, section 6 gives the experimental results \& discussion and finally section 7 gives the conclusion with future guidance.

\section{INSPIRING FACTORS}

Breast malignancy is the most well-known disease in ladies and long haul therapeutic viability of careful mediation relies upon precise staging of tumor malignant growth [1]. The size of tumor is the prime feature to forecast the breast cancer. Tumor size estimation is liable to potential blunders, and both tumor attributes and imaging constraints may differentially influence the estimation exactness of tests utilized for this reason. Underestimation of tumor size may in this manner lead to included surgical margins; overestimation may prompt excessively radical medical procedure (counting mastectomy when BCS may have been conceivable), and poorer psychosocial/ cosmetic results [2]. The modality which is frequently used for the breast radiologist's daily clinical practice is breast MRI. Several kinds of screening methods for breast cancer detection used are mammography, ultrasound, PET scan, MRI etc. Breast MRI is the most preferred diagnostic technique. Mammogram shows the contrast between bone density \& soft tissues while breast MRI produces crisp images \& explores even the small details of various breast tissues [2, 3]. It becomes a magnificent tool for breast diagnosis/screening for its advanced interpretation guidelines and imaging technique. The false positive rate of breast MRI is high as per its moderate specificity. However, studies have shown MRI to be superior to mammography, ultras onography, and clinical examination for revealing tumor extent in the breast. Furthermore, MRI can encourage radiologists and different authorities to decide how to treat breast malignancy patients by distinguishing the phase of the tumor. 
There are a few investigations contrasting these modalities' demonstrative execution, the breast MRI is the precise methodology to evaluate the tumor extent [4-7].

\section{III.RELATED WORK}

Forbes et al., (2006) [9] proposed a new image segmentation technique which consolidates Bayesian morphology and model-based clustering for the selection of ROI. Dataset of nineteen patients is used and results demonstrate that the technique segmented the image in an informative manner and also good in terms of detection rates.

Stoutjesdijk et al., (2007) [10] divided a breast lesion into a number of spatially adjacent clusters of comparative upgrade qualities using mean shift segmentation. This iterative method of classification distinguished the most suspicious cluster and finally the particular one represents the ROI of lesion. Such a ROI may be utilized in a CAD system for evaluating the attributes of contrast enhancement automatically.

Y. Ueda et.al. (2011) [11] proposed a new analysis method is to analyze the time-signal intensity curve (TIC) of each pixel and to extract ROI constructed from pixels with similar TIC. The study automatically determines the ROI with this new analysis method. The proposed technique provides better repeatability as well as comparative quantitative TIC analysis.

Stoutjesdijk et al., (2012) [12] presented a strategy for cluster-based investigation of energy enhancement in breast MRI. It gives an AUC (Area under curve) of 0.83 for the distinction between malignant \& benign lesion experimentally. It demonstrates that the iterative search method performs fundamentally superior for the most suspicious cluster than a non-iterative technique.

Chieh-Ling Huang (2014) [13] presented a shapemodel and LSM (level set method) to section breast lesions in MR images. The idea of ROI may be introduce to reduce the noise in various other breast regions. In the analysis, the proposed technique is contrasted with the Chan-Vese technique to show the efficacy of the presented technique. The exploratory outcomes demonstrate that the proposed work can do the effective segmentation of breast masses.

J. A. R. Ponnusamy \& C. N. K. Babu (2016) [14] proposed a new GSA-NRG (generalized simulated annealing and neutrosophic region growing) segmentation method for cancer detection through breast MRI. The outcomes demonstrated that this system enhances the True Positive Rate so the accuracy. It also limits the processing time adequately.

Jamil A. M. Saif et. al., (2016) [15] evaluated that the proposed method is highly effective for general as well as medical images like MRI/Endoscopic. Likewise demonstrated that the Canny edge detection is progressively powerful for the medical images where the tumor/abnormalities are less recognized from the background out of other algorithms.

\section{MATERIALS AND METHODS}

The proposed work introduces a quite robust but potent approach for cancer region detection specifically in breast
MRI which first detects the edges followed by the preprocessing techniques and then segments the inner and outer regions to find out the suspicious lesion. In the detection phase, filtering and edge detection technique is applied on input breast MR image. In segmentation phase, selection of peak \& valley points is done and fit to circle approach is applied to the selected points which outputs the outer boundary of the breast skin-line. For inner segmentation, morphological and hole-filling operations are performed. Fig. 1 described the outline of the algorithm with the following steps.

\section{A. Breast MRI dataset acquisition}

Initially, the images of breast MR are needed to load as the input data for further processing. Here in this paper, an online available S1 dataset [16] is used which contains approximately eighty multi-parametric MR images of breast tumor patients.

\section{Proposed Algorithm}

Input: $\mathrm{I}_{\mathrm{m}}(\mathrm{I}$ is breast MR Image and $\mathrm{m}=1,2,3 \ldots \mathrm{n})$ Output: $\mathrm{I}_{\mathrm{ER}}$ (Extracted Region Image)

1. Binaries the Query Image $I_{m}$ into $I_{B}$.

2. Identify $\mathrm{H}_{1 \mathrm{n}}, \mathrm{H}_{2 \mathrm{n}} \mathrm{V}_{\mathrm{n}}$ where ' $n$ ' is 1,2,3,4 all sides of image, $\mathrm{H}_{1}, \mathrm{H}_{2}$ and $\mathrm{V}$ are peak and valley point respectively.

3. $\mathrm{D}_{\mathrm{n}}=$ distance (Euclidian, $\mathrm{H}_{1 \mathrm{n}}, \mathrm{H}_{2 \mathrm{n}}$ and $\mathrm{V}_{\mathrm{n}}$ ).

4. Breast Region $\square \operatorname{Max}\left(D_{n}\right)$.

5. $\mathrm{I}_{\mathrm{BE}}$ - Remove Opposite (Breast region) from corner points $\mathrm{C}_{1}, \mathrm{C}_{2}$.

6. $\mathrm{I}_{\text {new }}-\mathrm{I}_{\mathrm{m}} * \mathrm{I}_{\mathrm{BE}}$

7. $\mathrm{I}_{\mathrm{BR}}-$ Brightest Region ( $\left.\mathrm{I}_{\text {new }}\right)$

8. $\mathrm{I}_{\mathrm{BRT}}$ - thinning $\left(\mathrm{I}_{\mathrm{BR}}\right)$

9. $\mathrm{I}_{\mathrm{ER}}$ - Largest Connected Component $\left(\mathrm{I}_{\mathrm{BRT}}\right)$

\section{B. $\quad$ Image enhancement}

It is a pre-processing step that plays an important role in image processing at low level. The hidden rule of preprocessing is to broaden the intensity level distinction among object and their background. It represents the breast lesions structure in a more reliable and proper manner. Fig 2 shows the filtering of breast MR image through Median and Wiener filtering techniques. In this, initially the original MR image is taken as input. Then impulse and Gaussian Noises are added with 0.05 \& 0.4 noise densities at stage 1 and calculate the statistical parameters i.e. MSE, PSNR, RMSE and MAE of noisy images respectively.

Retrieval Number: A2262109119/2019®BEIESP

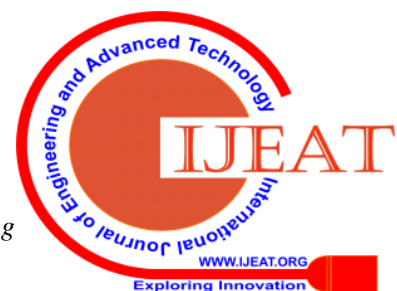


In Stage 2, Median filter is applied so obtained the filtered images along with the performance evaluation parameters. Similarly, Stage 3 gives the images filtered with wiener filter and also the statistical parameters of the filtered images are calculated.

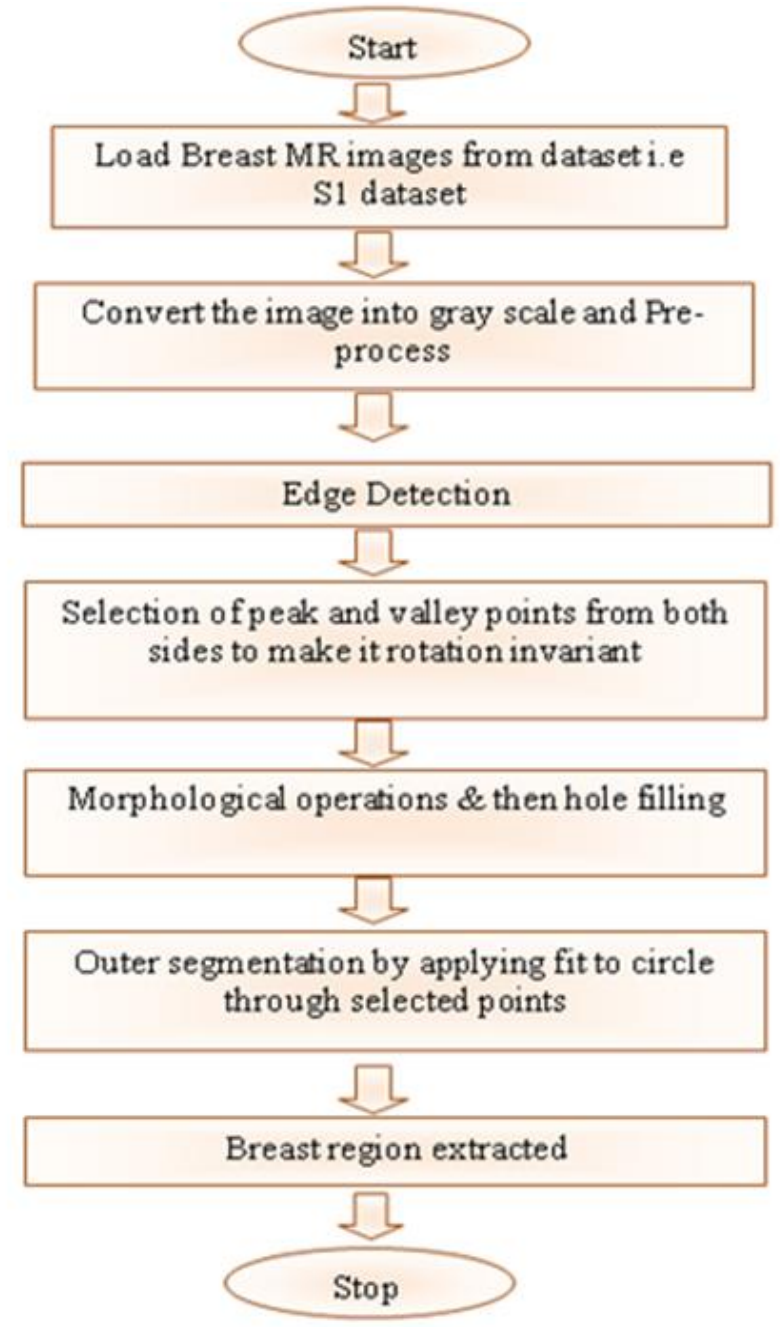

Fig. 1 Proposed methodology

\section{Apply edge detection technique}

An edge is a high frequency component at which abrupt changes take place corresponding to the features of image such as illumination, texture, contrast etc. The edges play an important role in extraction of ROI. There are various edge detection techniques which are currently available such as Roberts, Sobel, LOG, Prewitt edge detection techniques [17]. Here in this paper, canny operator is used for edge recognition as it gives more grounded edges when contrasted with other edge operators. The Canny operator is viewed as a standout amongst the best edge detectors as of now being used. Canny edge detection technique guarantees great noise reduction and in the meantime identifies genuine edge points with least error.

The accompanying criterion has optimized Canny as the edge detection method:

1) The high signal to noise ratio of the gradient further limits the probability of erroneously distinguishing nonedge points.
2) An edge location factor guarantees that the distinguished edge is confined as precisely as could be expected under the circumstances.

3) The chances of recognizing a single edge multiple times are nearly less.

\section{Selection of various points}

In this, some peak and valley points need to be selected termed as H1, H2 and V1 respectively shown in fig. 3(b). These peak points provided the upper boundary point whereas the valley point $\mathrm{V} 1$ given the mid-point of the right \& left breast. Similarly, the alternate points $\mathrm{H} 1$ ',H2' and V1' depicts the lower boundary points and the breast skinline boundary respectively so that the image can be well differentiated from other body parts mainly heart, liver ( viewed in the lower part of breast MR image along with some breast area). These selected peak and valley points from upper as well as lower side of the breast make the breast MR image rotation invariant. Generally, in the case of breast MRI, the images can be directed upward or downward. And sometimes, the images are shifted to any angle due to patients' movement. So, it may affect the decision of radiologist to find the exact location of tumor either in the form of angle or distance. Therefore, these problems can be overcome by making the breast MR image rotation invariant.

\section{E. Inner/Outer segmentation}

This is done by applying fit to circle operation/function to all the selected points i.e. peak as well as valley points through MATLAB. And finally the skin -line boundary of the breast region is segmented from the other body parts captured in the breast MRI screening tool shown in fig. 3(cd).

\section{F. Morphological \& hole-filling operations}

Furthermore in this step, two basic operations i.e. erosion and dilation [17] used in morphological image processing are taken into consideration. The erosion compresses the size of exact ROI (region of interest) as it removes the small details or shrinks the object to obtain the exact margins of the tumor. But the dilation bridged the gaps in order to regain the original shape/size of ROI. At this stage, the close loops are then highlighted with the help of imfill in built command of MATLAB that fills all the close loops present in the image. This operation also comes under the category of Morphological operations. Also the tumor region is extracted with exact shape via inner segmentation criteria depicted in fig. 3(f) clearly.

\section{V.PERFORMANCE ANALYSIS}

The methodology is implemented using MATLAB software and tested on S1 dataset [15] which contains eighty images. The steps i.e. hole-filling and morphological operations in which dilation is used to bridge the gaps between the skin-line boundaries of breast ; hole-filling filled the holes within the breast area ; and erosion is required to obtain the accurate tumor shape/size by the 
concise idea of mass/tumor. Fig. 3 depicts the entire process:

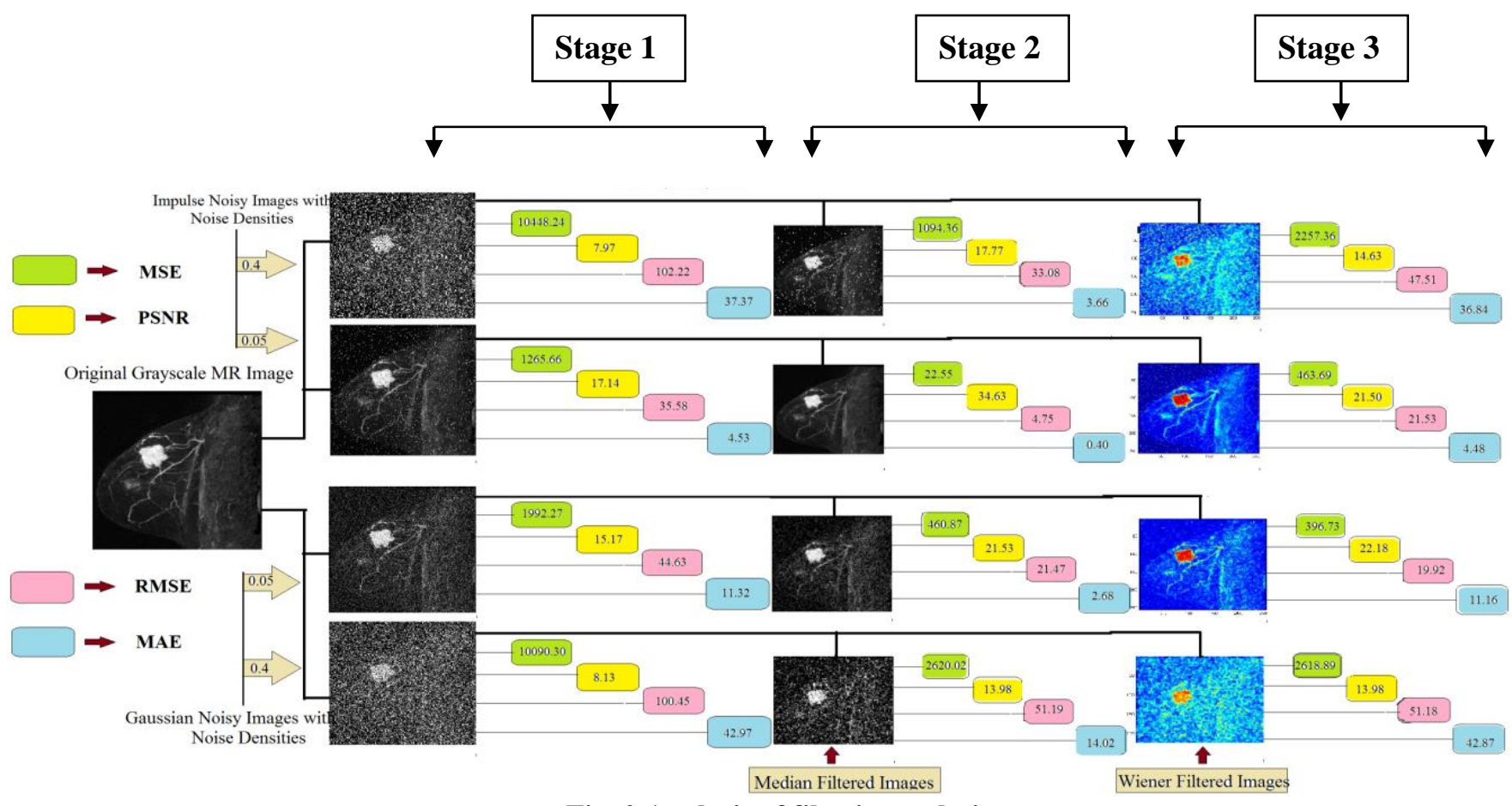

Fig. 2 Analysis of filtering technique

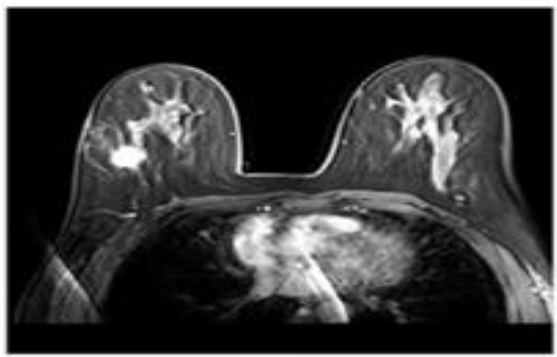

3 (a) Pre-processed Breast MR image

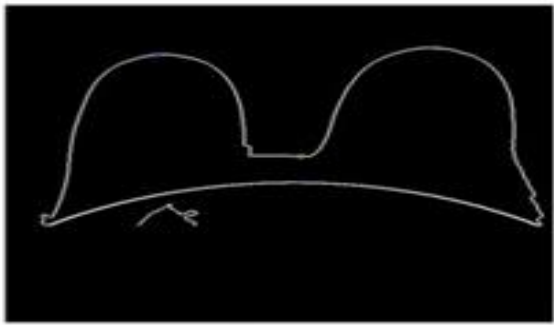

3 (c) Breast ROI extraction

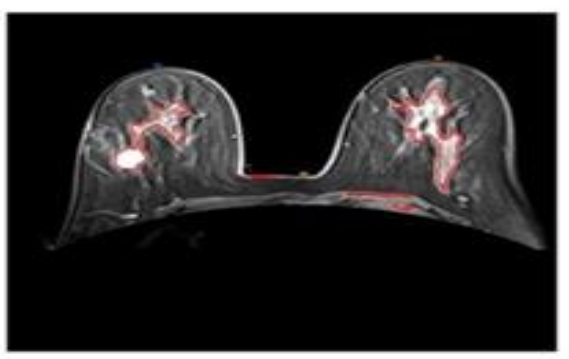

3 (e) Tissue segmentation

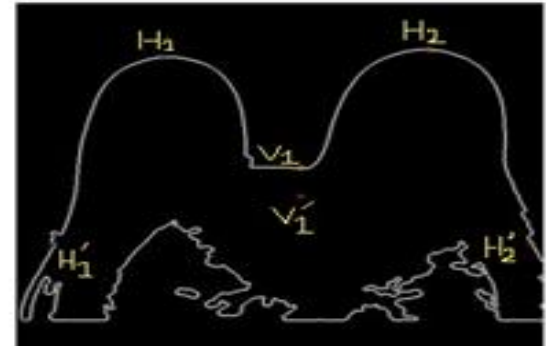

3 (b) Peak and valley point selection after edge detection through Canny

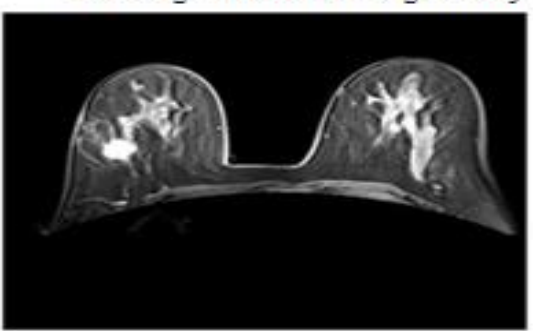

3 (d) Overlapped image

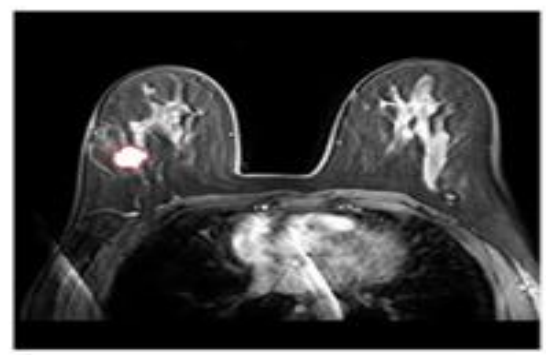

3 (f) Tumor extraction

Fig. 3 Tumor extraction process (a) Pre-processed Breast MR image (b) Peak and valley point selection after edge detection through Canny (c) Breast ROI extraction (d) Overlapped image (e) Tissue segmentation (f) Tumor extraction

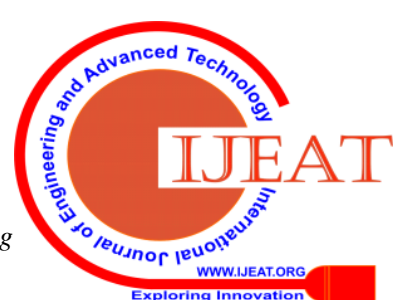




\section{VI.EXPERIMENTAL RESULTS AND DISCUSSION}

Initially, the breast MR image is loaded as input from the dataset and then Median and Wiener filters are applied to pre-process it as Fig. 2 shows. Thereafter, fig. 3(a) depicts the pre-processed breast MR image. The peak and valley points are further selected to segment the outer region of breast skin-line area and fig. 3(b) depicts those points clearly. The points are selected in such a way that it makes the breast MR region rotation invariant which is a unique feature among all the previous work performed in this field. The region boundary shown in fig. 3(c) is obtained through fit to circle function applying on the selected points. The morphological operation further did the inner segmentation to highlight the suspicious region of the inputted data. Visual analysis clears the fact that the proposed method efficiently detects as well as segments the suspicious legions from the input image as shown in fig. 3. Now, the binary segmentation of the tumor extracted image shown in fig. 3(f) is done to get the predicted image shown in fig 4(b) which needs to be compared with the ground truth/ reference one shown in fig. 4(a) (manually segmented by the radiologist/ expert) to find out the following parameters [18]:

Precision: The proportion of the points on the boundary line of predicted image lies nearby on the boundary points of the ground truth image to the length of the predicted boundary or the ratio of accurately predicted positive values to the total predicted positive ones.

\section{Precision $=T P / T P+F P$}

$>$ Recall (Sensitivity): The proportion of the quantity of points on the limit of the ground truth image those are close enough to the boundary of the predicted one to the length of the ground truth boundary or the ratio of positive observations those are correctly predicted to the total values.

$$
\text { Recall }=T P / T P+F N
$$

F1 Score: It computes the contour matching score i.e. Boundary F1 between the automatic segmentation in prediction and the manual segmentation in ground truth. It gives the weighted average of Recall \& Precision.

F1 score $=2 *($ Recall $*$ Precision $) /($ Recall + Precision $)$ Fig. 4 shows the manually and automatically segmented images:

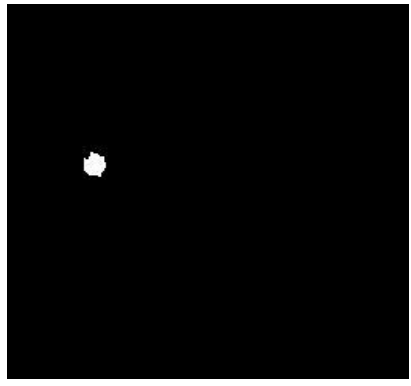

(a)

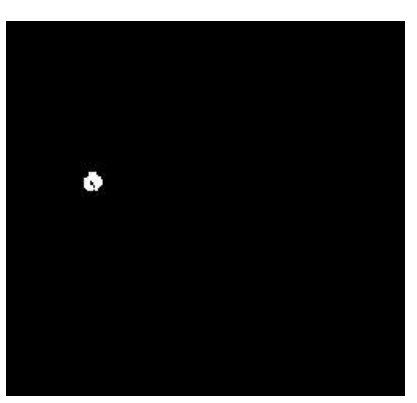

(b)
Table I Performance evaluation Parameters Performance Evaluation parameters (Radiologist vs. Automatic)

\begin{tabular}{|llll}
\hline & Score & Precision & Recall \\
\hline Image 1 & $85.00 \%$ & $75.00 \%$ & $100.00 \%$ \\
\hline Image 2 & $95.00 \%$ & $91.00 \%$ & $100.00 \%$ \\
\hline Image 3 & $100.00 \%$ & $100.00 \%$ & $100.00 \%$ \\
\hline Image 4 & $100.00 \%$ & $100.00 \%$ & $100.00 \%$ \\
\hline Image 5 & $100.00 \%$ & $100.00 \%$ & $100.00 \%$ \\
\hline Image 6 & $100.00 \%$ & $100.00 \%$ & $100.00 \%$ \\
\hline Image 7 & $100.00 \%$ & $100.00 \%$ & $100.00 \%$ \\
\hline Image 8 & $91.00 \%$ & $84.00 \%$ & $100.00 \%$ \\
\hline Image 9 & $100.00 \%$ & $100.00 \%$ & $100.00 \%$ \\
\hline Image 10 & $100.00 \%$ & $100.00 \%$ & $100.00 \%$ \\
\hline Average \% & $\mathbf{9 7 . 0 0 \%}$ & $\mathbf{9 5 . 0 0 \%}$ & $\mathbf{1 0 0 . 0 0 \%}$ \\
\hline
\end{tabular}

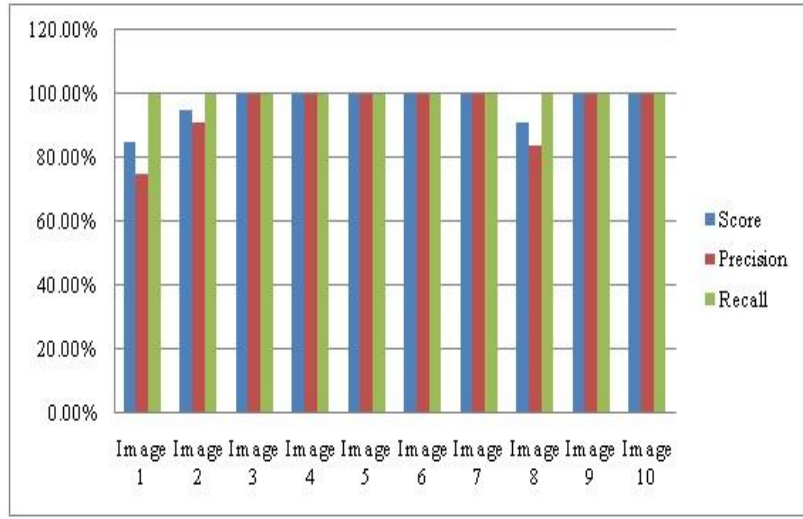

Fig. 5 Graphical representation of Statistical Parameters

\section{VII.CONCLUSION AND FUTURE WORK}

In this paper, median and wiener filters are used to remove salt \& pepper as well as Gaussian noise respectively. Then, Canny Edge Detection technique is used as a preprocessing step in order to extract the boundary. It gives the solid edges when contrasted with other operators and has conquered the issue of threshold alteration so the tumor extraction by utilizing morphological operations becomes more simple and effective. Finally, the manually segmented image done by the radiologist (ground truth) is statistically compared with the predicted image segmented automatically with the proposed algorithm. The performance evaluation matrices i.e. Score, Precision and Recall outcomes in the efficacy of the presented algorithm. The results considered as the average of the performance parameters for 10 cases. For future work, the proposed algorithm can be extended for further classification via selected features of the extracted region (suspicious) whether it is malignant or benign. Visual analysis depicts that the presented strategy is effective for inner as well as external segmentation. This is simple and quick as a result Fig. 4 (a) Manual Tumor segmentation (b) Automaticallyof utilizing fundamental techniques of image processing. It segmented tumor

Table I gives the qualitative results in the form of three performance evaluation parameters and its graphical representation given in Fig. 5. can likewise be useful in other applications of medical imaging, feature extraction and pattern recognition.

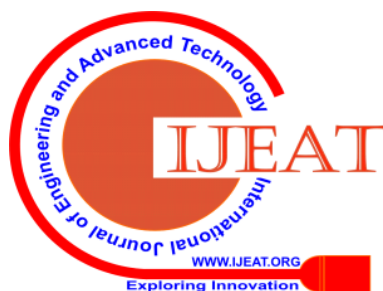


This method can be more adaptive if parameters' dependability be more flexible.

\section{REFERENCES}

1. Poonam Jaglan et. al., "Detection of Breast Cancer using MRI: A Pictorial Essay of the Image Processing Techniques" International Journal of Computer Engineering in Research Trends (IJCERT), vol. 6, issue 1, pp: 238-245, January, 2019.

2. Q. Al-Faris et al., "Breast MRI Tumour Segmentation using Modified Automatic Seeded Region Growing Based on Particle Swarm Optimization Image Clustering" 2012 online conference on soft computing in industrial applications anywhere on earth, pp. 1-11, December, 2012.

3. P. Jaglan, R. Dass, M. Duhan, " A Comparative Analysis of Various Image Segmentation Techniques", Proceedings of $2^{\text {nd }}$ International Conference on Communication, Computing and Networking, Lecture Notes in Networks and Systems, 46, https://doi.org/10.1007/978-98113-1217-5.36, Springer Nature Singapore Pte Ltd.

4. Uematsu T, Yuen S, Kasami M, Uchida Y. Comparison of magnetic resonance imaging, multidetector row computed tomography, ultrasonography, and mammography for tumor extension of breast cancer. Breast Cancer Res Treat, vol. 112, issue 3, pp: 461-74, 2008.

5. Berg WA, Gutierrez L, NessAiver MS, Carter WB, Bhargavan M, Lewis RS, et al. Diagnostic accuracy of mammography, clinical examination, US, and MR imaging in preoperative assessment of breast cancer. Radiology, vol. 233, issue 3, pp:830-49, 2004.

6. Bhooshan N, Giger ML, Jansen SA, Li H, Lan L, Newstead GM. Cancerous breast lesions on dynamic contrast-enhanced MR images: computerized characterization for image-based prognostic markers. Radiology, vol. 254, issue 3, pp:680-90, 2010.

7. Uematsu T, Kasami M, Yuen S. Comparison of FDG PET and MR for evaluating the tumor extent of breast cancer and the impact of FDG PET on the systemic staging and prognosis of patients who are candidates for breast-conserving therapy. Breast Cancer, vol. 16, issue 2, pp:97-104, 2009.

8. Rajeshwar Dass, Priyanka, Swapna Devi, "Image Segmentation Techniques," International Journal of Electronics \& communication Technology(IJECT), vol. 3, issue 1, pp. 66-70, March 2012.

9. Forbes et. al., "Model-based Region-of-interest Selection in Dynamic Breast MRI” J Comput Assist Tomogr, vol. 30, no. 4, pp. 675-687, 2006.

10. Stoutjesdijk et al., "Automated Analysis of Contrast Enhancement in Breast MRI Lesions Using Mean Shift Clustering for ROI Selection" Journal of magnetic resonance imaging, vol. 26, pp. 606-614, 2007.

11. Y. Ueda et.al., "Automatic ROI Determination by Analyzing Timesignal Intensity Curve in Dynamic Contrast Enhanced MR Imaging of the Breast", European society of radiology, C-1822, ECR, 2011.

12. Stoutjesdijk et al., "“'Computer Aided Analysis of Breast MRI Enhancement Kinetics Using Mean Shift Clustering and Multi-feature Iterative Region of Interest Selection" Journal of magnetic resonance imaging, vol. 36, pp. 1104-1112, 2012.

13. Chieh-Ling Huang, "Breast Mass Segmentation On Breast MRI Using The Shape-Based Level Set Method", Biomedical Engineering: Applications, Basis and Communications, Vol. 26, No. 4, 2014.

14. Joe Arun Raja Ponnusamy and C. Nelson Kennedy Babu, "Breast Lesion Segmentation Using Generalised Simulated Annealing and Neutrosophic Region Growing Algorithm in Breast MRI", Academic Journal of Cancer Research, vol.9(4), pp: 75-81, 2016.

15. Jamil A. M. Saif et. al., " Gradient Based Image Edge Detection", International Journal of Engineering and Technology, Vol. 8, No. 3, June 2016.

16. Thakran S, Chatterjee S, Singhal M, Gupta RK, Singh A, "Automatic Outer and Inner Breast Tissue Segmentation using Multi-Parametric MRI Images of Breast Tumor Patient”, PLoS ONE 13(1): e0190348, 2018. https://doi.org/10.1371/journal.pone.0190348

17. Rajeshwar Dass, Vikas, "Comparative Analysis of Threshold Based, K-mean and Level Set Segmentation Algorithms", International Journal of Computer Science \& Technology, vol. 4, issue 1, March 2013.

18. Rajeshwar Dass, Vikas, Priyanka, "Image Segmentation Performance Evaluation Methods," International Journal of Science, Engineering \& Computer Technology (IJSECT), vol. 2, Issue 1, pp. 125-127, March 2012.

\section{AUTHORS PROFILE}

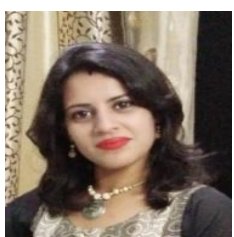

Poonam Jaglan received her B.Tech degree in Electronics \& Communication Engineering from Kurukshetra University in 2005 and M.Tech degree in 2010 from NCCE, Israna. Presently, she is pursuing her $\mathrm{PhD}$ from Deenbandhu Chhotu Ram University of Science \& Technology, Murthal. She has nine years of teaching experience. Her area of interest includes digital image processing, medical image processing and computer vision. Email: jaglanpoonam@gmail.com

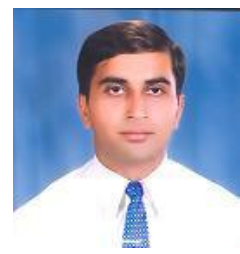

Dr. Rajeshwar Dass received his $\mathrm{PhD}$ from Deenbandhu Chhotu Ram University of Science \& Technology, Murthal in 2014 with specialization in image processing. He received his M.E Degree in ECE from National Institute of Technical Teachers Training and Research Center (NITTTR) Chandigarh in the year 2007. He received his B.E (Honors) Degree in ECE from Apeejay College of Engineering, Sohna, Gurgaon in 2004. In August 2004 he joined Dept. of ECE of Apeejay College of Engineering, Sohna, Gurgaon. Presently he is working as assistant professor in the department of Electronics \& Communication Engineering, DCRUST, Murthal since 2008. His interest includes Medical Image Processing, Neural Network, Wireless Communication and Soft Computing Techniques. He has contributed more than 40 technical papers in International Journals and Conferences. He has written books on wireless communication and basics of electronics.

Email: rajeshwardas10@gmail.com

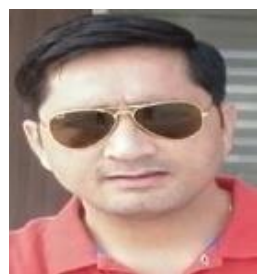

Manoj Duhan is Professor in ECE Department at DCRUST, Murthal (Haryana), India. He chaired the ECE department from 2008 to 2013 and from 2013 to 2017 the Biomedical Engineering Department ran under his chairmanship. Now again he is the chairman of ECE department of the same university. He was Reader and Chairman, ECE Department GJUST, Hisar from 2006 to 2008. He obtained his BE in Electronics and Telecommunication Engineering from NMU, Jalgaon in 1997, ME in Electronics and Communication Engineering from NIT, Kurukshetra in 2001 and PhD in Electronics Engineering from MDU, Rohtak. He has published more than 100 research papers. His current areas of interest are biomedical engineering, optimisation techniques, reliability and VLSI.

E-mail: duhan_manoi@rediffmail.com 